\title{
A molecular synchrotron
}

\author{
CYNTHIA E. HEINER ${ }^{1 \dagger}$, DAVID CARTY ${ }^{1 *}$, GERARD MEIJER ${ }^{1}$ AND HENDRICK L. BETHLEM ${ }^{1,2 \dagger}$ \\ ${ }^{1}$ Fritz-Haber-Institut der Max-Planck-Gesellschaft, Faradayweg 4-6, D-14195 Berlin, Germany \\ ${ }^{2}$ Laser Centre Vrije Universiteit, De Boelelaan 1081, NL-1081HV, Amsterdam, The Netherlands \\ *Present address: Physical and Theoretical Chemistry Laboratory, South Parks Road, Oxford OX1 3QZ, UK \\ †e-mail: heiner@fhi-berlin.mpg.de; rick@fhi-berlin.mpg.de
}

Published online: 21 January 2007; doi:10.1038/nphys513

Many of the tools for manipulating the motion of neutral atoms and molecules take their inspiration from techniques developed for charged particles. Traps for atoms-akin to the Paul trap for ions 1 - have paved the way for many exciting experiments, ranging from ultra-precise clocks ${ }^{2}$ to creating quantum degenerate matter ${ }^{3,4}$. Surprisingly, little attention has been paid to developing a neutral particle analogue of a synchrotron-arguably, the most celebrated tool of the chargedparticle physicist ${ }^{5,6}$. So far, the few experiments dealing with ring structures for neutral particles have used cylindrically symmetric designs $^{7-9}$; in these rings, no force is applied to the particles along the longitudinal direction and the stored particles are free to fill the entire ring. Here, we demonstrate a synchrotron for neutral polar molecules. A packet of ammonia molecules is accelerated, decelerated and focused along the longitudinal direction ('bunched') using the fringe fields between the two halves of a segmented hexapole ring. The stored bunch of cold molecules $(T=0.5 \mathrm{mK})$ is confined to a $3 \mathrm{~mm}$ packet even after a flight distance of over $30 \mathrm{~m}$ (40 round trips). Furthermore, we show the injection of multiple packets into the ring.

In traps, electromagnetic fields are used to keep particles confined in a region of space where they can be studied in complete isolation from the (hot) environment. In its simplest form, a storage ring is a trap in which the particles - rather than having a minimum potential energy at a single location in space-have a minimum potential energy on a circle. Storage rings such as these have been demonstrated for neutrons ${ }^{7}$, atoms ${ }^{8}$ and molecules ${ }^{9}$. The advantage of a storage ring over a trap is that packets of particles with a nonzero mean velocity can be confined. While circling the ring, these particles can be made to interact repeatedly, at well-defined times and at distinct positions with electromagnetic fields and/or other particles. To fully exploit the possibilities offered by a ring structure, it is imperative that the particles remain in a bunch as they revolve around the ring. This ensures a high density of stored particles, moreover, this makes it possible to inject multiple-either co-linear or counter propagating - packets into the ring without affecting the packet(s) already stored.

To provide the necessary forces to bunch the molecules, the cylindrical symmetry of the ring must be broken; as a consequence, the transverse confining force will vary as a function of the longitudinal position in the ring. The variation of the confinement force has major consequences for the stability of the particles's trajectories. For certain longitudinal velocities, the disturbance caused by the longitudinal focusing elements ('bunchers') will add up, meaning that all trajectories become unstable and those particles will be lost. These unstable velocity regions are known as 'stop bands' in charged-particle accelerator physics ${ }^{5,6}$. To minimize the width of these bands, corrective elements ('correcters') need to be implemented. Here, we demonstrate a molecular synchrotron based on a simple scheme to incorporate these necessary elements proposed by Crompvoets et al. ${ }^{10}$. A more elaborate design for a synchrotron was published by Nishimura et al. ${ }^{11}$. In a different approach, Murch et al. ${ }^{12}$ observed a reduced dispersion of ultracold atoms propagating at velocities close to a stop band in an atomic storage ring.

Our synchrotron, shown in Fig. 1a, consists of two hexapoles bent into a semicircle separated by a $2 \mathrm{~mm}$ gap. By switching the voltages applied to the electrodes, as shown in Fig. 1b-e, the necessary fields are created for confinement, to carry out stopband correction, for acceleration/deceleration and bunching, and to extract laser-ionized molecules, respectively. Figure if shows a time sequence for a round trip. In normal operation, the voltages are applied (as in Fig. 1b) such that the resulting electric field is zero at the centre and increases linearly radially outwards. Molecules in states that have a positive energy shift in the applied electric field-so-called low-field seekers-will experience a force towards the centre of the hexapole. In a curved hexapole, the molecules also experience a centrifugal force that depends on their forward velocity. At a certain radius, the inward force due to the Stark potential and the outward centrifugal force will cancel. For example, a deuterated ammonia $\left(\mathrm{ND}_{3}\right)$ molecule in the $|J, K\rangle=|1,1\rangle$ low-field-seeking state flying with a forward velocity of $87 \mathrm{~m} \mathrm{~s}^{-1}$ will be displaced radially outwards by $2 \mathrm{~mm}$ from the geometric centre of the hexapole. As this hypothetical molecule revolves around the ring, it will form a closed orbit that remains at this radial position. Molecules flying with the same forward velocity but with a different radial position or with a nonzero radial velocity will oscillate around this closed orbit with a frequency of around $600 \mathrm{~Hz}$.

In the gap region, the molecules can be both accelerated/ decelerated and bunched by applying the voltages shown in Fig. 1d to one bend, while the other bend is kept at ground potential. As molecules enter the gap, they are exposed to a field gradient that causes the molecules to gain Stark energy and consequently lose kinetic energy, that is, the molecules are decelerated. As the molecules exit the gap, however, they are exposed to an opposing field gradient that causes the molecules to lose Stark energy and thus gain kinetic energy, that is, the molecules are accelerated. The time sequence is synchronized to the position (and velocity) of one hypothetical molecule, appropriately referred to as the 'synchronous' molecule, assuring that this molecule is always at the same position in the gap when the fields are switched. It will, 

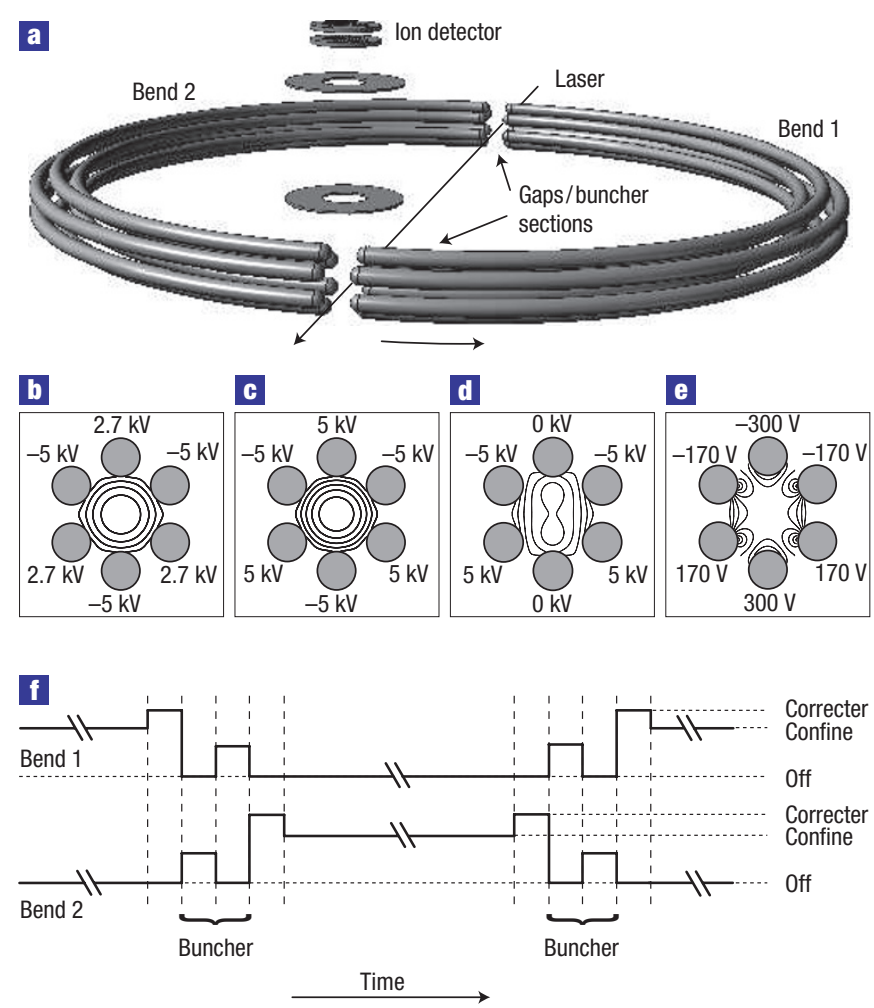

Figure 1 Scheme of the synchrotron. a, Experimental set-up. $\mathrm{ND}_{3}$ molecules with a velocity of $87 \mathrm{~m} \mathrm{~s}^{-1}$ (kinetic energy of $6.33 \mathrm{~cm}^{-1}$ ) are tangentially injected into bend 1 of the segmented hexapole ring. The ring radius is $12.5 \mathrm{~cm}$ with two $2 \mathrm{~mm}$ gaps between the two halves; the diameter of the electrodes is $4 \mathrm{~mm}$. Molecules in the detection region are ionized using so-called $(2+1)$-resonance enhanced multiphoton ionization with pulsed laser light around $317 \mathrm{~nm}$, and are then extracted perpendicularly to the plane of the ring and counted by an ion detector. $\mathbf{b}-\mathbf{e}$, Voltage configurations necessary for confinement (b), performing stop-band correction (c), acceleration/deceleration and bunching (d) and extracting laser-ionized molecules (e). The contours represent the absolute electric field in steps of $8 \mathrm{kV} \mathrm{cm}^{-1}$ for $\mathbf{b}-\mathbf{d}$, and of $0.2 \mathrm{kV} \mathrm{cm}^{-1}$ for $\mathbf{e . ~ f , ~ T i m e ~ s e q u e n c e ~ f o r ~ m o l e c u l e s ~ c o m p l e t i n g ~ o n e ~ r o u n d ~ t r i p ~}$ (without detection), starting from the lower most arrow indicated in a.

therefore, lose or gain the same amount of kinetic energy per round trip. It follows that molecules in front of the synchronous molecule will lose more kinetic energy than the synchronous molecule. Vice versa, molecules that are behind the synchronous molecule will gain more kinetic energy than the synchronous molecule. As a result, the molecules within a small position and velocity interval will experience a force towards the synchronous molecule and will oscillate around it; the molecules are trapped in a travelling potential well that revolves around the ring. This concept is known as phase stability ${ }^{13,14}$ and forms the basis of all modern chargedparticle accelerators and storage rings. An extensive discussion of the application of phase stability for decelerating polar molecules in so-called Stark decelerators can be found elsewhere ${ }^{15,16}$.

In the gap, the molecules are not transversely confined. We compensate for this by increasing the focusing force during a short period of time before and after the molecules pass through the gap, by switching to the correcter configuration shown in Fig. 1c. For detection, the stored molecules are ionized and extracted perpendicularly to the plane of the ring, using the configuration shown in Fig. 1e, and then counted by an ion detector.

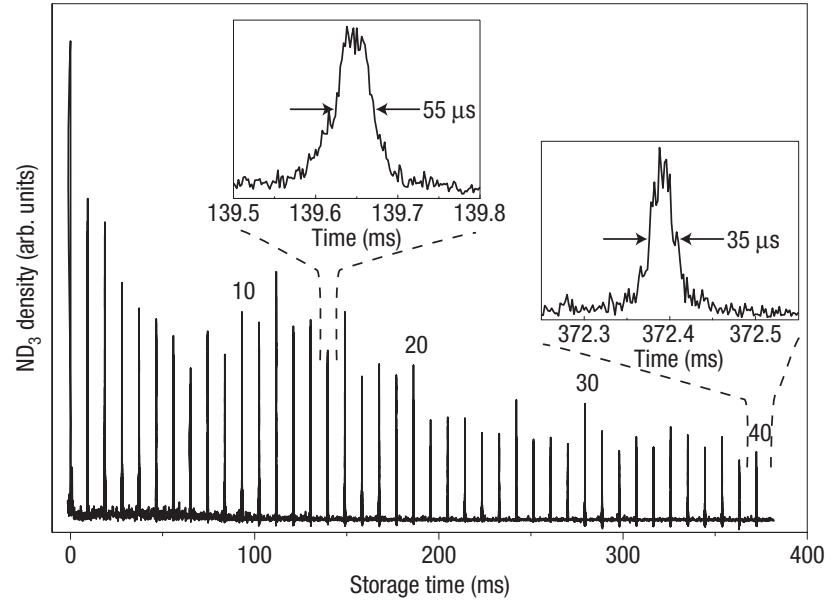

Figure 2 Round-trip TOF profiles. The density of ammonia molecules at the detection zone inside the synchrotron as a function of storage time up to the fortieth round trip, currently limited only by our electronics. Zoom-ins of two TOF profiles are shown as insets, more clearly illustrating the absolute widths of these peaks. From gaussian fits to these profiles, we determine for the fifteenth round trip a temporal width of $55 \mu \mathrm{s}$, corresponding to a position spread of $4.8 \mathrm{~mm}$, and for the fortieth round trip a temporal width of $35 \mu \mathrm{s}$, corresponding to a position spread of $3.0 \mathrm{~mm}$.

In our experiments, the synchrotron is injected with packets of Stark-decelerated ammonia molecules. Both the mean value and the width of the velocity distribution of the injected beam is tuneable; details of the injection beam line can be found elsewhere ${ }^{17}$. Figure 2 shows the ammonia density in the detection zone of the ring as a function of storage time. The velocity of the stored packet is kept at $87 \mathrm{~m} \mathrm{~s}^{-1}$, implying that the molecules take about $9.3 \mathrm{~ms}$ to make one round trip in the $81-\mathrm{cm}$-circumference ring. After an initial rapid decrease, and some modulations, the signal is seen to become relatively constant. Starting from about the twentieth round trip, the signal decays with a 1/e time of $0.5 \mathrm{~s}$, consistent with losses due to collisions with background gas in our $2 \times 10^{-8}$ mbar vacuum.

The time-of-flight (TOF) profile for each round trip shown in Fig. 2 was fitted to a gaussian. From these fits, we infer the position spread of the stored packet in the detection region, plotted as circles in Fig. 3a. The width of the packet is seen to decrease until about the twentieth round trip, after which it stabilizes around a value of $3 \mathrm{~mm}$. To determine the longitudinal temperature of the packet in the ring, we have measured the expansion rate of the packet in the absence of the buncher. The inset of Fig. 3a shows the expansions when the molecules are released from the longitudinal well after the fourth and twenty-fifth round trip, indicated by triangles and squares, respectively. The solid lines in the inset show the results from a simple formula fitted to the expansions. We find the velocity spread of the packet released after the fourth round trip to be $1.7 \mathrm{~m} \mathrm{~s}^{-1}$, corresponding to $1.3 \mathrm{mK}$, and after the twenty-fifth round trip to be $1.1 \mathrm{~m} \mathrm{~s}^{-1}$, corresponding to $0.5 \mathrm{mK}$. Although initially confined, the hottest molecules are slowly expelled owing to coupling between the longitudinal and transverse motions, causing the initial decrease in the position spread and temperature. As both the width and the temperature of the packet stay constant for the later round trips, we conclude that the trajectories of these molecules are stable and they will, in principle, be confined indefinitely.

From the gaussians fitted to the data shown in Fig. 2, we also determine the time needed by the molecules to complete one 

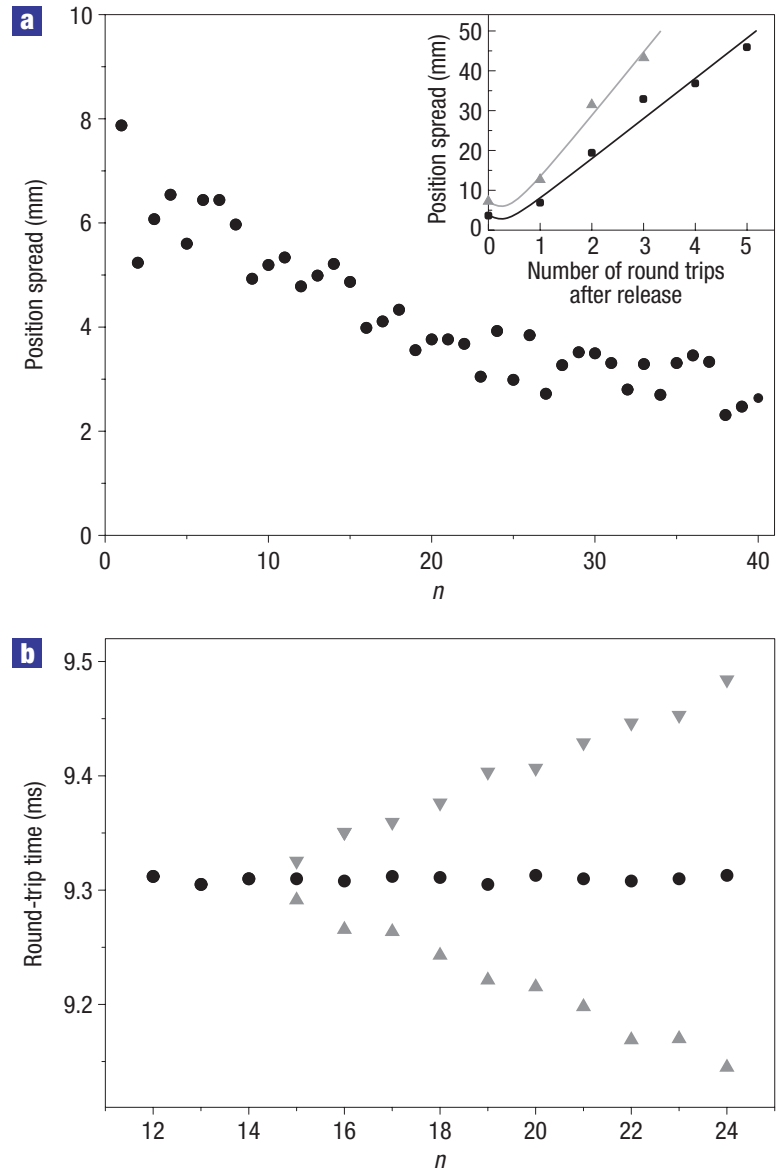

Figure 3 Analysis of individual round trips. a, The position spread of the stored molecules in the detection zone, determined from the TOF profiles shown in Fig. 2, plotted as a function of the number of completed round trips, $n$. The inset shows the expansion of the molecules when they are released after the fourth (triangles) and twenty-fifth (squares) round trips. The solid lines in the inset show the results from a simple fit $\Delta z(t)=\left(\Delta z_{0}^{2}+\left(\Delta v_{z} \times\left(t-t_{0}\right)\right)^{2}\right)^{1 / 2}$, where $\Delta z$ is the position spread, $\Delta v_{z}$ is the velocity spread, $t_{0}$ is the time at which the packet has its minimum position spread (at the quarter-ring position) and $\Delta z_{0}$ is the position spread at that time. $\mathbf{b}$, The round-trip time, defined as $t(n)-t(n-1)$, where $t$ is the time of arrival and $n$ is the number of completed round trips, plotted as a function of $n$. Measurements are shown when the mean velocity of the packet is held constant (circles), accelerated (triangles) or decelerated (down-triangles).

round trip; these times are plotted as circles in Fig. 3b. In these measurements the velocity is held constant, and therefore the round trip time is also constant. We have also conducted measurements in which, starting after the fifteenth round trip, we change the molecules' kinetic energy by $0.025 \mathrm{~cm}^{-1}$ per round trip. The arrival time of the accelerated and decelerated packets, indicated in Fig. 3b by up-triangles and down-triangles, respectively, are plotted as a function of round-trip time.

Independent control over each half ring also enables the injection of multiple molecular packets into the ring without affecting the packet(s) that are already stored. Figure 4 shows a similar measurement to that in Fig. 2, where we now inject a second packet $95.3 \mathrm{~ms}$ after injection of the first packet. To bunch both packets simultaneously, the fields are switched twice as often, that is, we introduce a 'fake' gap at a quarter-ring position. In this case, the second packet trails the first by $20 \mathrm{~cm}$. In principle, we

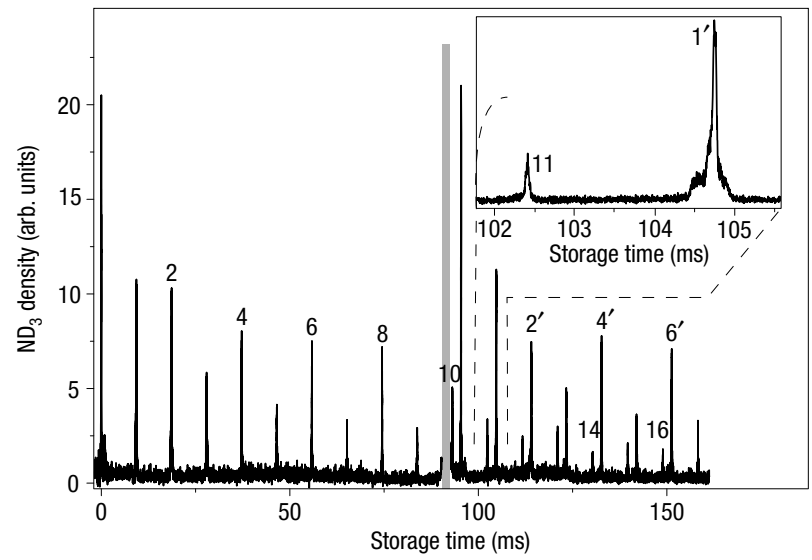

Figure 4 Multiple packets in the ring. TOF profiles showing two packets of ammonia molecules revolving inside the synchrotron. The second packet is injected $95.3 \mathrm{~ms}$ after the first packet, and trails it by $20 \mathrm{~cm}$. Before injection of the second packet, the detection system is briefly switched off (indicated by the grey bar) to avoid saturation of the detector as the undecelerated part of the ammonia beam passes through the detection region. The alternating intensities observed arise from a less-than-ideal loading of the packet into the ring. The inset shows a zoom-in of a TOF profile of the first packet after completing its eleventh round trip and the second packet after completing its first round trip.

can load many more packets into the ring by introducing more 'fake' gaps. However, as molecules in a 'fake' gap experience a different transverse force to that experienced in a true gap, a more promising route is to construct a ring out of many short segments. As the symmetry of such a ring is higher, the transverse well will be deeper. Moreover, as the depth of the longitudinal well is inversely proportional to the distance between the gaps, the longitudinal well will also be deeper.

Trapped molecules are being considered for various precision tests and collision studies (an overview can be found in ref. 18). For many of these applications, confining molecules in a ring rather than in a more conventional trap offers many advantages ${ }^{10}$. One of the most obvious applications is to use the ring as a neutral molecule collider. A stored packet of molecules revolving around the ring will meet counter-propagating packets. By storing many packets over an extended time, the sensitivity for detecting collisions increases by orders of magnitude. For instance, in a ring containing 10 packets revolving in both directions, a packet having completed 100 round trips will have had 2,000 encounters. As the velocity of the beam is tuneable, the collision cross-section can be measured as a function of beam energy, and resonances in the collision complex can be recorded-a low-energy molecularphysics experiment as the ultimate replica of high-energy nuclearphysics experiments.

Received 16 November 2006; accepted 13 December 2006; published 21 January 2006.

References

1. Paul, W. Electromagnetic traps for charged and neutral particles. Rev. Mod. Phys. 62, 531-540 (1990).

2. Ido, T. \& Katori, H. Recoil-free spectroscopy of neutral Sr in the Lamb-Dicke regime. Phys. Rev. Lett. 91, 053001 (2003).

3. Cornell, E. A. \& Wiemann, C. E. Bose-Einstein condensation in a dilute gas, the first 70 years and some recent results. Rev. Mod. Phys. 74, 875-893 (2002).

4. Ketterle, W. When atoms behave as waves: Bose-Einstein condensation and the atom laser. Rev. Mod. Phys. 74, 1131-1151 (2002).

5. Humphries, S. Jr. Principles of Charged Particle Acceleration (Wiley, New York, 1986).

6. Lee, S. Y. Accelerator Physics 2nd edn (World Scientific, Singapore, 2004).

7. Kügler, K.-J., Paul, W. \& Trinks, U. A magnetic storage ring for neutrons. Phys. Lett. B 72, 422-424 (1978). 
8. Sauer, J. A., Barrett, M. D. \& Chapman, M. S. Storage ring for neutral atoms. Phys. Rev. Lett. 87, 270401 (2001)

9. Crompvoets, F. M. H., Bethlem, H. L., Jongma, R. T. \& Meijer, G. A prototype storage ring for neutra molecules. Nature 411, 174-176 (2001).

10. Crompvoets, F. M. H., Bethlem, H. L. \& Meijer, G. A storage ring for neutral molecules. Adv. At. Mol. Opt. Phys. 52, 209-287 (2005).

11. Nishimura, H., Lambertson, G., Kalnins, J. G. \& Gould, H. Feasibility of a synchrotron storage ring for neutral polar molecules. Rev. Sci. Instrum. 74, 3271-3278 (2003).

12. Murch, K. W., Moore, K. L., Gupta, S. \& Stamper-Kurn, D. M. Dispersion management using betatron resonances in an ultracold-atom storage ring. Phys. Rev. Lett. 96, 013202 (2006).

13. Veksler, V. I. A new method for acceleration of relativistic particles. J. Phys. (USSR) 9 , 153-158 (1945).

14. McMillan, E. M. The Synchrotron—a proposed high energy particle accelerator. Phys. Rev. 68, 143-144 (1945).

15. Bethlem, H. L., Crompvoets, F. M. H., Jongma, R. T., van de Meerakker, S. Y. T. \& Meijer, G. Deceleration and trapping of ammonia using time-varying electric fields. Phys. Rev. A 65 , 053416 (2002)

16. Gubbels, K., Meijer, G. \& Friedrich, B. Analytic wave model of Stark deceleration dynamics. Phys. Rev. A 73, 063406 (2006).
17. Heiner, C. E., Bethlem, H. L. \& Meijer, G. Molecular beams with a tunable velocity. Phys. Chem. Chem. Phys. 8, 2666-2676 (2006).

18. Bethlem, H. L. \& Meijer, G. Production and application of translationally cold molecules. Int. Rev. Phys. Chem. 22, 73-128 (2003).

\section{Acknowledgements}

We thank F. M. H. Crompvoets for help in the early stages of the project and for discussions. We acknowledge the technical assistance of A. J. A. van Roij and H. Haak and design and construction of the electronics by G. Heyne. This work was supported by the EU-network on 'Cold Molecules'.

D.C. acknowledges support from the ESF Network on Collisions in Atom Traps (CATS).

H.L.B. acknowledges financial support from the Netherlands Organisation for Scientific Research

(NWO) via a VENI-grant.

Correspondence and requests for materials should be addressed to C.E.H. or H.L.B.

Competing financial interests

The authors declare that they have no competing financial interests.

Reprints and permission information is available online at http://npg.nature.com/reprintsandpermissions/ 